\title{
A general psychopathology factor in early adolescence
}

Praveetha Patalay, Peter Fonagy, Jessica Deighton, Jay Belsky, Panos Vostanis and Miranda Wolpert

\section{Background}

Recently, a general psychopathology dimension reflecting common aspects among disorders has been identified in adults. This has not yet been considered in children and adolescents, where the focus has been on externalising and internalising dimensions.

\section{Aims}

To examine the existence, correlates and predictive value of a general psychopathology dimension in young people.

\section{Method}

Alternative factor models were estimated using self-reports of symptoms in a large community-based sample aged 11-13.5 years $(N=23477)$, and resulting dimensions were assessed in terms of associations with external correlates and future functioning.

\section{Results}

Both a traditional two-factor model and a bi-factor model with a general psychopathology bi-factor fitted the data well. The general psychopathology bi-factor best predicted future psychopathology and academic attainment. Associations with correlates and factor loadings are discussed.

\section{Conclusions}

A general psychopathology factor, which is equal across genders, can be identified in young people. Its associations with correlates and future functioning indicate that investigating this factor can increase our understanding of the aetiology, risk and correlates of psychopathology

\section{Declaration of interest}

None.

\section{Copyright and usage}

(c) The Royal College of Psychiatrists 2015.
The latent structure underpinning psychiatric diagnoses has been a subject of renewed interest. ${ }^{1-4}$ In particular, the existence of similar correlations and comorbidity between disorders and symptoms in adult populations has led to recent re-examinations of the dimensionality of psychopathology in two key studies, one of 18- to 64-year-olds ${ }^{1}$ and the other of participants in a longitudinal study repeatedly assessed at age 18,21, 26, 32 and $38 .^{2}$ Both concluded that a hierarchical model fitted the data best, one in which (1) all the various dimensions loaded either onto distinct externalising, fears and distress ${ }^{1}$ or internalising and externalising ${ }^{2}$ dimensions at one level, yet (2) onto a single dimension at another level, resulting in it being labelled a 'hierarchical' or 'bi-factor' model. Caspi and associates ${ }^{2}$ refer to the higher-order dimension as 'general psychopathology'. The results of both studies indicate that general psychopathology better represents a longitudinal risk of psychiatric disorders and suggest that studying this factor will allow for a better understanding of the aetiology, correlates and prognosis of psychiatric disorder. The risk factors examined in these studies indicate that external correlates are linked with the internalising, externalising and thought disorder dimensions, primarily because they are associated with general psychopathology. The only exception was gender, which was not associated at all with general psychopathology. ${ }^{2}$

In child psychopathology, two main dimensions have been widely used for decades to characterise the structure of mental health in individuals below the age of 18: most commonly referred to as the internalising and externalising spectra. ${ }^{5,6}$ Although the dimensions are associated moderately, with studies indicating correlations between 0.4 and $0.6,{ }^{7,8}$ they have been regarded as distinct and key dimensions of child mental health difficulties and most research findings are considered in relation to these two distinct dimensions. Evidence supports the existence of both age- and gender-related patterns in relation to these dimensions; thus, externalising disorders are more likely to be early onset and afflict males, whereas adolescence and female gender are characterised by a greater prevalence of internalising symptoms. ${ }^{9}$

In light of this gender and developmental distinction, it seems conceivable that a general psychopathology factor might not characterise child mental health. To address this issue, the current research examined the structure of psychopathology in a large community-based paediatric sample substantially younger than those included in the aforementioned adult work, namely 11- to 13.5-year-olds. The goal was to ascertain whether a general psychopathology dimension could be identified in early adolescence. Moreover, we sought to evaluate the external validity and relevance of a hierarchical model that includes general psychopathology by exploring associations with sociodemographic predictors, educational correlates and social competencies in adolescence. ${ }^{10}$ Additionally, we assessed the ability of the derived dimensions to predict future psychopathology and academic functioning, thereby determining the predictive utility of a general, bi-factor dimension of psychopathology.

\section{Method}

\section{Participants}

Data were collected from 23477 participants (representing a response rate of approximately $73 \%)$ studying in Year 7 (65.7\%, $N=15433)$ and Year $8(34.3 \%, N=8044)$ across 210 statemaintained secondary schools in England as part of a national study of mental health and provision in schools, the Me and My School study. Details of the wider study are published elsewhere. ${ }^{11}$ Of the participants $50.4 \%$ were female and mean age was 12.05 years $($ s.d. $=0.56$ ), with $99.9 \%$ of the sample ranging from 11 to 13.5 years of age. The sample was predominantly White (76.2\%), followed by Asian (8.7\%), Black (5.9\%), Mixed (3.8\%) and other ethnicities (1.4\%); $4 \%$ were unclassified. Almost a fifth 
(19.7\%) were eligible for free school meals, which serves as a proxy for economic deprivation. ${ }^{12}$ In comparison with all school students in England in the same year (White $81.9 \%$, eligible for free school meals $15-17 \%),{ }^{13}$ sample characteristics indicate higher proportions of deprivation and ethnic minority groups in the current sample (a finding mainly explained by the inclusion of only state-maintained schools, as privately funded fee-paying schools were not included in the wider study).

Follow-up data were available for:

(a) Psychopathology: 1 year later, a sub-sample of 10270 participants (mean age 13.07 years (s.d. $=0.56$ ), female $51.6 \%$, eligible for free school meals $18.5 \%$, White $76.5 \%$ ) from 124 schools completed the mental health measures at a follow-up assessment. Attrition was mainly because of entire schools not participating in the follow-up. However, follow-up sample characteristics indicate that attrition was biased, with significantly $(P<0.05)$ more boys and deprived participants being lost to follow-up.

(b) Academic attainment: a sub-sample of 7569 participants (representing 94\% of eligible participants) who were in Year 8 at time 1 had standardised national test scores a year and a half later, obtained from national records. This sub-sample consisted of $54.4 \%$ female, $16.6 \%$ eligible for free school meals and $77.6 \%$ White, indicating that students without attainment scores were significantly more likely to be male, deprived and from ethnic minorities.

\section{Procedure}

At both time points, computer-based mental health surveys were completed by pupils within the normal school day with support provided by teachers. More specifically, teachers read to the class standardised information, including details about the study, confidentiality of responses and the right not to participate or to drop out at any time. Data on sociodemographic characteristics such as gender, deprivation, ethnicity and age were obtained from the National Pupil Database, a centrally collated database that holds all education-related data on all pupils in England. Academic attainment scores in national standardised tests were also obtained from the National Pupil Database in following years. Ethics permission for data collection was granted by the University College London research ethics committee.

\section{Measures}

\section{Psychopathology}

Participants completed two questionnaires reporting on mental health symptoms, Me and My School and the Strengths and Difficulties Questionnaire (SDQ). Owing to the conceptual nature of the analysis, items from both measures that represented either internalising or externalising dimensions were used because both measures have their own distinct items and using multiple measures was judged to increase validity, especially as the factor analyses to be reported were not meant to represent structures of existing 'measures' but instead the 'constructs/dimensions' of psychopathology that they represent. The only similar item across the two measures was 'I worry a lot'; it was retained in both as they correlated only 0.55 across the two instruments, which probably resulted from the different response options of the two measures: the frequency-based response options of the Me and My School questionnaire and the endorsement-based options of the SDQ (see below).

Me and My School Questionnaire. Symptoms were measured using the 10-item emotional difficulties and the 6-item behavioural difficulties scales of the Me and My School Questionnaire. ${ }^{7}$ This community-based screening measure of mental health difficulties has relatively simple, easy-to-understand items and has been validated for use by children as young as 8 years. ${ }^{7}$ The measure has been demonstrated to have good content validity, internal reliability, construct validity and sensitivity; analyses indicate that items do not operate differently across sub-groups based on special educational needs (SEN), deprivation and English as additional language. ${ }^{7,14}$ Participants respond to each item by endorsing one of three response options: never, sometimes and always. ${ }^{7}$ Preliminary analysis revealed that one item, 'I am shy', belonging to the emotional difficulties scale loaded poorly onto the internalising scale (factor loading $=0.29$ ); this item was thus excluded from the final analysis. The internal reliability, Cronbach's alpha, was 0.78 for the emotional difficulties and 0.80 for the behavioural difficulties scales.

Strengths and Difficulties Questionnaire. The SDQ is a widely used self-report measure of child mental health and has been shown to have good construct validity and reliability. ${ }^{15}$ The 5 -item emotional symptoms and conduct problems scales were used in the present study. Participants respond to each item by endorsing one of three response options: not true, somewhat true and certainly true. Cronbach's alpha was 0.72 for the emotional symptoms and 0.66 for the conduct problems scales.

\section{Correlates}

The external variables considered in this study include gender, deprivation, educational attainment, SEN, peer problems and pro-social behaviour. Deprivation was measured using (a) free school meals eligibility, which is a binary indicator often used as a school-based proxy for deprivation, and (b) the Income Deprivation Affecting Children Index (IDACI), a variable representing the deprivation ranking of the neighbourhood in which a child lives. SEN was based on the extent of special educational provision for each student and had the values no SEN, school action, school action plus and statement of SEN. Academic attainment was assessed using standardised national assessment scores at age 11 (Key stage 2) averaged across English, mathematics and science, higher score indicating better attainment. Social competence-related constructs such as peer problems and pro-social behaviour were measured using the respective 5-item self-report scales of the SDQ, ${ }^{15}$ scores ranging from 0 to $10 .^{15}$

\section{Future functioning}

Psychopathology. Self-reported mental health symptoms in the follow-up wave a year on were used to classify caseness or not based on both the Me and My School and SDQ scores. This approach was taken to allow investigation of the prediction of possible future diagnosis and clinical need. Caseness for the SDQ total difficulties was based on the abnormal threshold score of 20. Me and My School emotional symptoms used the clinical threshold of 12 and behavioural symptoms score of $7,{ }^{7}$ with Me and My School overall caseness indicated by above threshold scores on either scale. For all the variables, individuals above threshold were coded ' 1 ' and below threshold ' 0 ' for the analysis. From the sub-sample of 10270 participants who completed the Me and My School and SDQ a year on, based on the Me and My School clinical threshold, $11.7 \%$ were classified as exhibiting caseness at this time-point, with $3.9 \%$ above threshold on the emotional difficulties and $9.4 \%$ on the behavioural difficulties scale. For the SDQ total difficulties, $7.3 \%$ had above threshold scores at follow-up. 
Poor academic attainment. National standardised test scores taken at the end of Year 9 in England, referred to as Key stage 3, were used as measures of academic functioning. Scores can range from 0 to 8 and according to government-set standards pupils are expected to achieve at least level $5 .{ }^{16}$ As outlined in the Participants section, Key stage 3 scores were available for the 7569 participants who were in Year 8 at time 1 . The variable was coded ' 1 ' for 1832 participants $(24.2 \%)$ who had achieved below level 5 , indicating poor academic functioning.

\section{Results}

Analyses were conducted in three stages: (1) to examine the structure of psychopathology and the possibility of a single, hierarchical dimension of general psychopathology factor in young people; (2) to evaluate the sociodemographic and educational correlates of the (i) general, (ii) internalising and (iii) externalising factors in the alternate factor solutions from stage 1; and (3) to determine the predictive value of the different dimensions by assessing the extent to which general psychopathology and the internalising and externalising dimensions predicted future psychopathology and academic functioning.

\section{Stage 1: The structure of psychopathology}

We utilised the standard approach to establishing hierarchical structured dimensions or constructs, ${ }^{17}$ which is similar to the approach used by Caspi et al. ${ }^{2}$ However, the measurement unit used differed from the two adult studies, ${ }^{1,2}$ in which symptomlevel scores (items) were utilised to define factors in the models rather than diagnosis-level scale scores. Confirmatory Factor Analysis (CFA) was conducted to test three different models: (a) a correlated factors model; (b) a bi-factor or hierarchical model; and (c) a one-factor model. The first model concerns the widely used two-factor (internalising and externalising) solution. The second model introduces the hierarchical dimension of a general psychopathology bi-factor, on which every variable loads, in addition to loading onto the internalising or externalising dimension that they represent. The third model is a one-factor model in which all items load onto a single factor; it evaluates the hypothesis that the single, one-factor model can account for variation without the need for specific lower-order internalising and externalising factors. Results from Caspi et $a l^{2}$ suggest that this third model is not a good fit to the data; given the established nature of internalising and externalising factors in child psychopathology, we expect this model not to fit the data well.

CFAs were estimated in Mplus $7{ }^{18}$ and the weighted least square means and variances (WLSMV) estimator was used as it is the most suited for categorical manifest variables. ${ }^{19}$ Model fit was assessed using the comparative fit index (CFI), Tucker Lewis index (TLI) and root mean square error of approximation (RMSEA). CFI and TLI closer to 1 indicate good fit to the data and RMSEA closer to 0 indicates good fit. The values were judged by the widely used conservative criteria of CFI and TLI $>0.95$ and RMSEA $<0.06$ to indicate a very good model fit. ${ }^{20}$ Owing to the non-nested nature of the models, models could not be compared directly using a difference in fit statistic (e.g. Akaike information criterion) as they cannot be estimated when WLSMV estimation is used. Hence, model fit criteria and factor loadings were used to assess the quality of models.

\section{Model 1: Two-factor model with internalising} and externalising dimensions

The first CFA tested the commonly employed two-factor model with separate internalising and externalising dimensions. The model allows for the internalising and the externalising factors to be correlated. The first columns in online Table DS1 present the model fit statistics and standardised factor loadings for this model. As might be expected, factor loadings of the items on the two factors were all positive, above 0.5 and significant $(P<0.001)$, indicating that the two-factor model explains the data reasonably well. Moreover, model fit statistics revealed that the model fit was acceptable; RMSEA was 0.06 and CFI (0.93) and TLI (0.93) were just under the accepted threshold for very good model fit (0.95).

\section{Model 2: Bi-factor model with a general psychopathology bi-factor}

The second model was a bi-factor model with internalising and externalising as lower-order factors and general psychopathology as a higher order bi-factor; this model assumes that the derived factors are not correlated (Fig. 1). ${ }^{21}$ This model fitted the data well $(\mathrm{CFI}=0.95, \mathrm{TLI}=0.94$, RMSEA $=0.05$; see online Table DS1 for factor loadings). Loadings on the general factor were all moderate and significant $(P<0.001)$, with an average factor loading of 0.43 . However, in this model loadings on internalising were not all high and significant, with both items relating to sleep ('I wake up in the night'; 'I have problems sleeping') having zero order loadings $(0.07,0.0002)$ on the internalising-specific factor. This suggests that these two items more directly predict general psychopathology rather than internalising. Factor loadings on the externalising factor were all moderate to high and significant $(P<0.001)$, with an average factor loading of 0.59 (Fig. 1).

\section{Model 3: One-factor model}

The one-factor model assigned each item only to a general overall factor. Model fit statistics indicate that this model did not fit the data well $(\mathrm{CFI}=0.7, \mathrm{TLI}=0.68$, RMSEA $=0.12$, see online Table DS1 for factor loadings). Factor loadings on the one-factor were moderately high and significant $(P<0.001)$, with an average loading of 0.57 .

To summarise, the first set of analyses indicate that the hierarchical bi-factor solution explains the data best in terms of model fit statistics, although the traditional internalising-externalising two-factor model proved almost as good. The one-factor model did not explain the data well, clearly suggesting that a general psychopathology factor on its own does not sufficiently represent these data. Therefore, subsequent analysis involving established correlates/predictors of psychopathology focused only on factors from the first two models. For this purpose, factor scores were outputted from both models (1 and 2).

Correlations between these factor scores from both models were estimated. In the two-factor model the correlation between internalising and externalising was moderate $(0.45, P<0.001)$, whereas in the bi-factor model internalising and externalising correlated negatively and more weakly $(-0.16, P<0.001)$. The general psychopathology bi-factor correlated $0.30(P<0.001)$ with internalising and $0.22(P<0.001)$ with externalising scores in the bi-factor model.

\section{Stage 2: Associations with external correlates}

Factor scores from both models were correlated with variables with established associations with psychopathology in childhood in order to assess the external validity of the dimensions while examining the relative associations of general psychopathology and the specific internalising and externalising factors with the external correlates (Table 1).

Inspection of Table 1 reveals that gender did not correlate significantly with general psychopathology in the bi-factor 


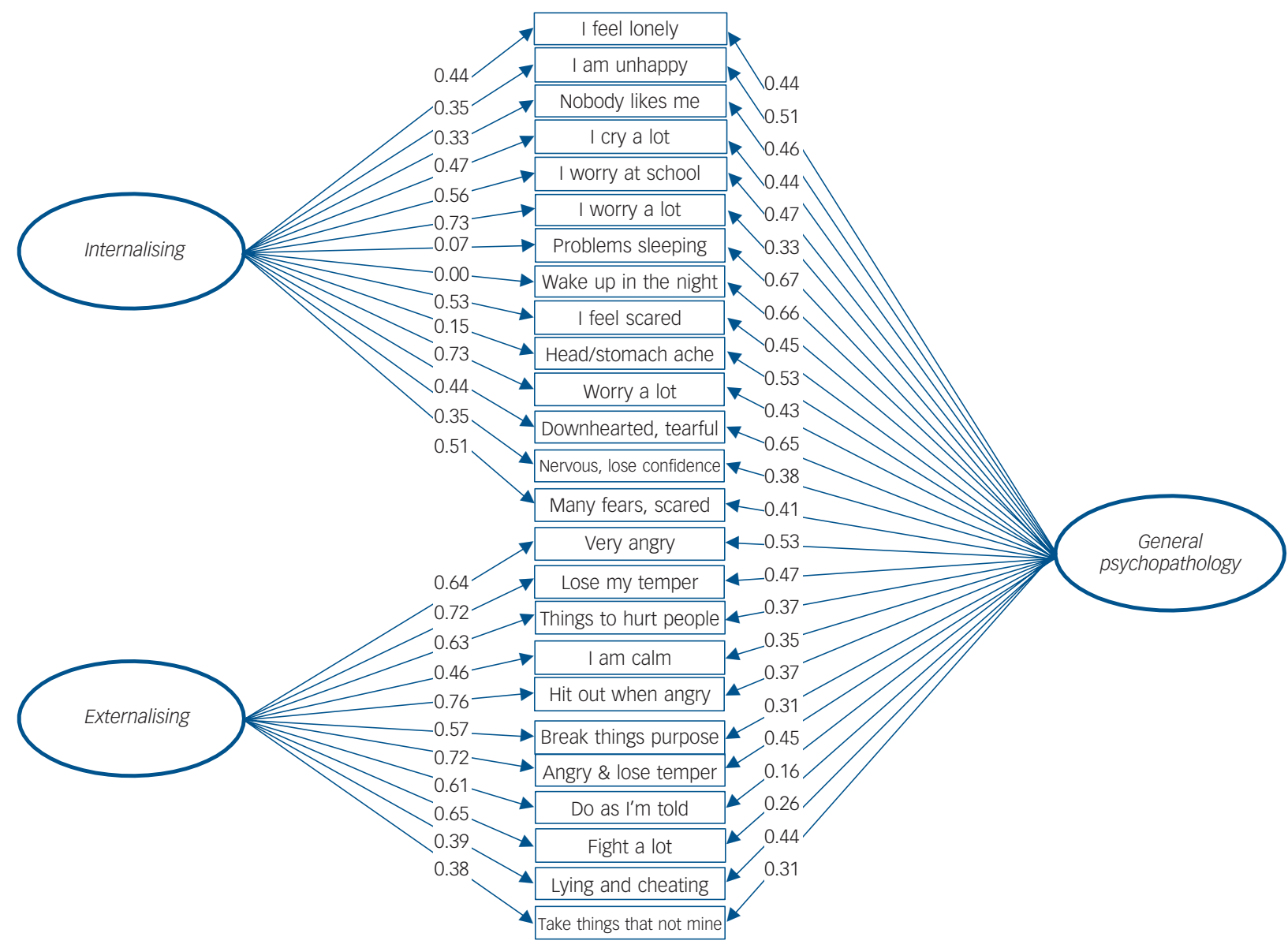

Fig. 1 Bi-factor model with the item loadings onto the internalising and externalising dimensions and the general psychopathology bi-factor.

model. Girls scored significantly higher, though, on internalising and significantly lower on externalising than boys in both the two- and bi-factor models. Correlations involving both indicators of social class, free school meals and IDACI, revealed that economic deprivation was related to higher levels of general psychopathology and greater externalising problems in both models. In the case of internalising, however, the significant association between internalising and the two socioeconomic status indicators proved positive in the two-factor model but negative in the bi-factor model; in absolute terms, however, these significant correlation coefficients did not differ by very much, as each was close to zero, proving significant as a result of the large sample size. Adolescents with SEN classifications scored higher on problems, however parameterised, in both models, with the reverse being true of adolescents who scored high on attainment. In the latter case, however, the association with internalising problems in the bi-factor model was not significant.

\section{Stage 3: Predicting future functioning}

To evaluate the predictive validity of the factor scores derived from the two-factor and bi-factor models, logistic regressions were conducted to predict future psychopathology caseness and poor academic attainment using factor scores from both models, while

\begin{tabular}{|c|c|c|c|c|c|}
\hline \multirow[b]{2}{*}{ Predictor } & \multicolumn{2}{|c|}{ Two-factor model (model 1) } & \multicolumn{3}{|c|}{ Bi-factor model (model 2) } \\
\hline & Internalising & Externalising & Internalising & Externalising & General psychopathology \\
\hline Gender $^{\dagger}$ (Female) & $0.13^{* *}$ & $-0.21 * *$ & $0.23^{* *}$ & $-0.27^{* *}$ & -0.007 \\
\hline $\mathrm{FSM}^{\dagger}(\mathrm{Yes})$ & $0.04^{* *}$ & $0.14^{* *}$ & $-0.02^{* *}$ & $0.14 * *$ & $0.08 * *$ \\
\hline IDACl & $0.02^{*}$ & $0.14 * *$ & $-0.05^{* *}$ & $0.14^{* *}$ & $0.08 * *$ \\
\hline SEN $^{\dagger}$ (Yes) & $0.10^{* *}$ & $0.14^{* *}$ & $0.03^{* *}$ & $0.11^{* *}$ & $0.13^{* *}$ \\
\hline Attainment & $-0.10 * *$ & $-0.20 * *$ & -0.001 & $-0.17^{* *}$ & $-0.14^{* *}$ \\
\hline Peer problems & $0.53^{* *}$ & $0.31 * *$ & $0.35^{* *}$ & $0.09 * *$ & $0.48^{* *}$ \\
\hline Pro-social behaviour & $-0.04^{* *}$ & $-0.37 * *$ & $0.10 * *$ & $-0.40^{* *}$ & $-0.14^{* *}$ \\
\hline
\end{tabular}




\begin{tabular}{|c|c|c|c|c|c|c|c|c|c|c|}
\hline \multirow[b]{2}{*}{ Predictor } & \multicolumn{2}{|c|}{$\begin{array}{c}\text { SDQ } \\
\text { total difficulties }\end{array}$} & \multicolumn{2}{|c|}{$\begin{array}{c}\text { Me and My School } \\
\text { overall }\end{array}$} & \multicolumn{2}{|c|}{$\begin{array}{c}\text { Me and My School } \\
\text { emotional }\end{array}$} & \multicolumn{2}{|c|}{$\begin{array}{c}\text { Me and My School } \\
\text { behavioural }\end{array}$} & \multicolumn{2}{|c|}{$\begin{array}{l}\text { Poor academic } \\
\text { attainment }\end{array}$} \\
\hline & B & OR & B & OR & B & OR & B & OR & B & OR \\
\hline \multicolumn{11}{|l|}{ Two-factor model } \\
\hline Internalising & $1.44^{* * *}$ & 4.23 & $0.60 * * *$ & 1.83 & $2.27 * * *$ & 9.65 & 0.04 & 1.04 & $0.29 * * *$ & 1.33 \\
\hline Externalising & $1.00 * * *$ & 2.72 & $1.38 * * *$ & 3.97 & $0.27 * *$ & 1.28 & $1.72^{\star \star *}$ & 5.58 & $0.57 * * *$ & 1.76 \\
\hline \multicolumn{11}{|l|}{ Bi-factor model } \\
\hline Internalising & $0.97 * \star *$ & 2.63 & $0.24 *$ & 1.27 & $1.61^{\star * *}$ & 5.00 & -0.19 & 0.83 & $0.28^{* *}$ & 1.32 \\
\hline Externalising & $0.95^{* \star *}$ & 2.59 & $1.39 * \star \star$ & 4.04 & 0.08 & 1.08 & $1.82^{\star \star \star}$ & 6.16 & $0.65^{\star * *}$ & 1.91 \\
\hline General psychopathology & $2.84^{* * *}$ & 17.05 & $2.31 * * *$ & 10.08 & $3.02^{* * *}$ & 20.54 & $1.98^{* * *}$ & 7.25 & $0.85^{\star * *}$ & 2.34 \\
\hline
\end{tabular}

controlling for sociodemographic correlates including gender, SES and ethnicity (results in Table 2). From the bi-factor model the regression coefficients indicated that all three predictors significantly and positively predicted future psychopathology, measured with both the Me and My School and SDQ. Odds ratios (ORs) for factor scores from the bi-factor model predicting future psychopathology were as follows: ORs for the general psychopathology factor predicting future caseness were $>10$ (Me and My School, OR = 10.08; SDQ, OR =17.05), ORs for internalising factor were 1.27 and 2.63 and for externalising factor were 4.04 and 2.59 for the Me and My School and SDQ respectively. Factor-specific scores from the two-factor internalising-externalising model predicted future psychopathology with ORs of 1.83 and 4.23 for the internalising factor and 3.97 and 2.72 for the externalising factor. In the case of predicting specific emotional or behavioural symptoms, the general psychopathology factor significantly predicted future emotional problems $(\mathrm{OR}=20.54)$ and behavioural symptoms $(\mathrm{OR}=7.25)$.

Regressions predicting future academic functioning indicate that in the bi-factor model the general psychopathology factor $(\mathrm{OR}=$ $2.34)$, externalising $(\mathrm{OR}=1.91)$ and internalising $(\mathrm{OR}=1.32)$ significantly predicted future attainment. Both the internalising $(\mathrm{OR}=1.33)$ and externalising $(\mathrm{OR}=1.76)$ dimensions from the two-factor model significantly predicted future academic functioning.

Based on an effect size interpretation, where $\mathrm{OR}=6.71$ is considered a large effect, ${ }^{22}$ general psychopathology predicting future psychopathology can be considered large effects, with most other domains having small or medium predictive capacity.

\section{Discussion}

Recent years have witnessed a resurgence of interest in trying to characterise the dimensionality of adult psychopathology. ${ }^{3,4}$ The presence of a general psychopathology bi-factor was recently examined in adults by Lahey et $a l^{1}$ and Caspi et al. ${ }^{2}$ Both investigations indicate that this general psychopathology factor was associated with known external correlates of disorder that predict future psychopathology. As the youngest participants in both of these studies were 18 years of age, we sought to determine whether a similar psychopathology structure would emerge with a paediatric sample. We also examined the external correlates and predictive validity of dimensions of psychopathology discerned. Results of each set of analyses are discussed in turn, followed by a discussion of implications and future directions.

\section{A general psychopathology dimension in young people}

Results with our younger sample proved very much in line with those emerging from the two studies of older individuals.
Thus, we also found that a hierarchical model reveals a higherdimension general psychopathology factor over and above the classic two-factor, internalising and externalising dimensions. Investigation of the bi-factor model and the traditional two-factor model yielded some interesting insights into the structures and relationships between these dimensions (or disorder liabilities). With regard to associations between internalising and externalising dimensions, the traditional two-factor model yielded a correlation of 0.45 , which is in-line with the known moderate association between these dimensions. However, in the bi-factor model, internalising and externalising correlated negatively, clearly suggesting that previously identified associations are mainly explained by a common underlying factor. After removing variance associated with general vulnerability to psychopathology, internalising and externalising emerge as distinct yet unrelated styles of expressing psychopathology with potentially unique relationships with other demographic and clinical characteristics.

Importantly, the factor loadings in the different models result in clearer understanding of the externalising and internalising dimensions at the symptom level once the general common vulnerability to psychopathology is removed. For instance, sleep disturbance emerges as a poor indicator of internalising problems and might be better conceived of as a generic indicator of vulnerability to psychiatric disorder. All the other items, in most cases, represent the general factor to a certain extent and also the specific factor they are expected to represent. Considering the different age-group and geographical location of the current sample compared with the two studies that focused on adult psychopathology, ${ }^{1,2}$ as well as the use of symptom-level rather than diagnosis-level variables in the current investigation, the findings reported here clearly buttress the claim emerging from the prior work that there exists a general psychopathology factor, which is now discernible from at least the beginning of the second decade of life.

\section{External correlates}

Examination of some sociodemographic and educational correlates of the dimensions in the two models further illuminated differences between them. In the two-factor internalisingexternalising model all the examined correlates were associated significantly with the two dimensions, as would be expected from known associations of these variables in young people. ${ }^{10}$ This situation changed, however, once the general psychopathology dimension, was taken into account.

Considering first the case of internalising problems, the internalising dimension had reduced associations with SEN and previously positive associations between internalising and indices of socioeconomic deprivation are close to zero and negative once 
general psychopathology was taken into account; moreover, associations with educational attainment completely disappeared. The last finding might to some extent explain why research examining associations between internalising and education have been largely inconclusive. ${ }^{23}$ The results from the bi-factor model suggest that internalising problems are associated with educational and social problems only to the extent that they are linked with a general vulnerability to psychiatric disorder. The unique contribution of internalising problems, although significant in a large population-based study, is almost negligible in terms of prediction of attainment. This finding, if shown to be robust in future studies, may have significant policy implications.

The externalising dimension, once general psychopathology was taken into account, retained positive associations with the deprivation variables. Similarly, with general psychopathology accounted for, externalising retained negative associations with educational attainment. These findings indicate that characteristics of externalising problems might be linked uniquely to deprivation and impact on learning and attainment, even after a general propensity to psychopathology is taken into account.

In the current study, gender was not associated with general psychopathology, a finding similar to that reported in at least one of the prior studies of general psychopathology in adults. ${ }^{2}$ Additionally, after variance associated with general vulnerability was controlled, associations between gender and the internalising and externalising dimensions increased. This suggests that the gender specificity of the internalising and externalising spectra frequently observed in epidemiological studies ${ }^{10}$ may be stronger than previously thought, at least once overall vulnerability to mental disorder is separately identified; if replicated, this result will be important in terms of understanding development and morbidity in psychopathology. Gender has been a variable of much focus in the study of childhood psychopathology, to the extent that it has become common practice to study single-sex samples ${ }^{24}$ for both internalising and externalising disorders. In doing so, the study of comorbid symptoms and other risk factors quite often takes a back seat to gender in studies of developmental psychopathology. The general propensity for psychopathology might actually be equal across the genders. Although it remains important to understand the differential inclinations to developing certain kinds of disorders, which are likely to result from a multiplicity of factors (e.g. hormones, ${ }^{25}$ evolutionary pressures ${ }^{9}$ ), the finding that gender is not associated with a general liability to psychopathology suggests a need to reconsider traditional approaches to studying gender and psychopathology, including the role of gender in research on the aetiology and development of disorder. The findings reported here suggest that gender should be a less important part of efforts to understand a general liability to psychopathology, yet an important focus in models of specific disorders and their associated correlates.

Correlations with peer problems and pro-social behaviour provide insight into how these scales might be related to internalising or externalising dimensions. The analysis suggests that, even when general psychopathology is accounted for, prosocial behaviours are correlated with the externalising dimension. On the other hand, peer problems are associated most with general psychopathology rather than internalising, contrary to expectations based on existing research with the SDQ. ${ }^{26}$

\section{Predicting future functioning}

In terms of predictive value, the general psychopathology bi-factor significantly predicted odds of future psychopathology with large effect sizes, whereas the internalising and externalising dimensions had small or moderate predictive effect sizes. These results are consistent with similar findings in the prior studies of older individuals and suggest that utilising the general psychopathology dimension can, as Lahey et al (p. 77) ${ }^{1}$ observed, 'substantially improve prognostic predictions of future psychopathology and functioning', beginning as early as the second decade of life. General psychopathology also significantly predicted future academic attainment, indicating that the construct has predictive utility beyond psychopathology in other key domains of young people's functioning.

\section{Limitations and future directions}

It has been suggested that the general psychopathology dimension emerging in this and prior work might be a statistical artefact. ${ }^{2}$ However, evidence of external validity and predictive utility from this and the two existing adult studies clearly suggest otherwise and support wider implications and applications of this bi-factor dimension. Lahey and colleagues ${ }^{1}$ note the possibility that the correlations between different disorder symptoms might in part be because of biases in reporting based on implicit theories of psychopathology. They suggest that some individuals experiencing one symptom might also report other symptoms based on expectations of symptoms rather than their actual occurrence. ${ }^{1}$ In child psychopathology research the practice of collecting proxy reported symptoms might to an extent help evaluate this hypothesis, although it is likely that proxy reporters might also demonstrate these biases. In any case, replicating the analyses and results reported herein with proxy reported child psychopathology data is necessary, helping perhaps to advance understanding of the high levels of disagreement between reporters of child psychopathology. ${ }^{27}$

The current study is limited in the conclusions that can be drawn because the items included in the measurement instruments do not represent the full range of psychopathology symptoms experienced in childhood and adolescence. However, as recognised in both the adult studies, ${ }^{1,2}$ and even more pertinent to child studies, is the lack of population-level datasets that have measures across all possible diagnostic categories of psychopathology. Replication with a variety of different samples and a more comprehensive set of measures would be one way of further validating and increasing our understanding of the general psychopathology dimension or liability towards disorder.

The existence of the general psychopathology dimension, which represents commonalities across various disorders, suggests that future research into the aetiological factors, biological markers, environmental risk factors and expression of psychopathology will benefit from pooling together resources and having a more unified approach to studying general psychopathology, replacing or complementing present practice in which individual disorders are the primary focus of attention. This could increase our efficiency in identifying and understanding the different factors and their interplay that result in psychiatric disorder. There is already much evidence that supports this route. The most compelling data can be found in the consistent empirical observation that environmental and demographic risk factors associated with most types of diagnosis/disorders are similar rather than disorder specific. This is further supported by recent genetic shared risk models from twin studies ${ }^{28}$ and studies of gene loci associations of psychopathology ${ }^{29}$ which indicate that the biological correlates of major psychiatric disorders are similar. Additionally, partitioning out and studying disorders in their 'purer' forms after accounting for general psychopathology could enhance the identification of biomarkers and correlates that differentiate one disorder from another, if these are indeed unique. 
Finding a general psychopathology factor at a younger age provides support for the hypothesis that diagnostic specificity increases with age. Relative to adult disorders, childhood disorders tend to be less clear-cut vis-a-vis diagnostic criteria (e.g. anxiety and depression are less distinct in young people), which is one of the reasons the broader classifications of internalising and externalising dimensions have been long established and successfully employed in child psychopathology research. ${ }^{2}$ The possibility of liability to general psychopathology even in childhood suggests that disorder specificity increasing with age could be a result of gradually increasing tendencies to express psychopathology in particular ways. It will be of interest to determine whether a general psychopathology factor would emerge in research on children during the elementary or even preschool years.

Caspi et $a l^{2}$ also note that although thought disorders are a distinct third category in adult psychopathology, they are absent in the study of child psychopathology. In the current study the two variables related to difficulties in sleeping loaded exclusively onto general psychopathology which suggests that these variables might be indicators of a precursor to the thought disorder dimension in adulthood. However, the item on somatic symptoms (headaches, stomach aches, sickness) also loaded predominantly on general psychopathology rather than internalising, which suggests an alternative possibility that they, along with the sleep items, might provide a more organic or psychosomatic aspect of general psychopathology, which is reflected in their separate categorisation in diagnostic schemas. It is a limitation of the current study that the primary measures used were developed with a two-factor model of psychopathology in mind. In various preliminary models of instruments, cross-loading items between the two spectra of externalising and internalising may well have been selectively eliminated to improve fit. Future studies may need to create instruments deliberately designed to capture the hierarchical bi-factor model.

Mental health treatment for the most part has a different approach from most physical health treatment in that diagnosis and treatment are largely dependent on symptoms exhibited rather than the root or cause of the disorder. To illustrate the potential usefulness of further exploring this general psychopathology propensity, especially in terms of clinical utility and treatment, parallels can be drawn with immuno-deficiencies in physical health. Immunocompromised individuals are more susceptible to infections and the type and nature of the diseases/ infections that individuals with these deficiencies experience vary. ${ }^{30}$ Identifying the immunodeficiency rather than just observing the expressed symptoms is crucial to the long-term management and treatment of individuals with these immune deficits. Similarly, the general psychopathology factor places the emphasis on considering the individual's inherent propensity for psychopathology, rather than simply focusing on diagnostic categories and symptoms. This would take into consideration a person's developmental history, previous psychopathology and the current manifestation of symptoms and would involve evaluating an individual's propensity alongside the factors that might have triggered a particular set of symptoms. The existence of a general psychopathology factor or deficit suggests that individuals with greater propensity (or higher general psychopathology) are more likely to experience psychopathology no matter what, with environmental factors and life events perhaps serving only as moderators and triggers of the expression of specific disorders. If this is the case, as has been suggested, ${ }^{2}$ individuals with higher general psychopathology would be expected to transition through different diagnostic categories throughout their lifetime. ${ }^{2}$ There is already some evidence that this might be the case, both in childhood and in adulthood, ${ }^{31}$ and this hypothesis can be explicitly tested in longitudinal studies of psychopathology.

\section{Funding}

Research funded by the Department for Children, Schools and Families (now the Department for Education), England.

\section{Acknowledgements}

We thank the other members of and advisors of the TaMHS research group who were involved in the wider project and the staff and students of participating schools for their contributions to this research.

Praveetha Patalay, MPhil, Evidence Based Practice Unit (EBPU), University College London, and the Anna Freud Centre, London, UK; Peter Fonagy, PhD, FBA, FMedSCi, University College London, London, UK; Jessica Deighton, PhD, Evidence Based Practice Unit (EBPU), University College London, and the Anna Freud Centre, London, UK; Jay Belsky, PhD, Department of Human Ecology, University of California, Davis, California, USA; Panos Vostanis, MB, MD, FRCPsych, The Greenwood Institute of Child Health, Leicester University, Leicester, UK; Miranda Wolpert, DClinPsychol, Evidence Based Practice Unit (EBPU), University College London, and the Anna Freud Centre, London, UK

Correspondence: Praveetha Patalay, EBPU, University College London and the Anna Freud Centre, 21 Maresfield Gardens, London NW3 5SU, UK. Email: praveetha.patalay.11@ucl.ac.uk

First received 7 Apr 2014, final revision 11 Nov 2014, accepted 11 Nov 2014

\section{References}

1 Lahey BB, Applegate B, Hakes JK, Zald DH, Hariri AR, Rathouz PJ. Is there a general factor of prevalent psychopathology during adulthood? J Abnormal Psych 2012; 121: 971-7.

2 Caspi A, Houts RM, Belsky DW, Goldman-Mellor SJ, Harrington H, Israel S, et al. The $p$ factor: one general psychopathology factor in the structure of psychiatric disorders? Clin Psychol Sci 2014; 2: 119-37.

3 Kotov R, Ruggero CJ, Krueger RF, Watson D, Yuan Q, Zimmerman M. New dimensions in the quantitative classification of mental illness. Arch Gen Psych 2011; 68: 1003-11.

4 Wright AG, Krueger RF, Hobbs MJ, Markon KE, Eaton NR, Slade T. The structure of psychopathology: toward an expanded quantitative empirical model. J Abnormal Psych 2013; 122: 281-94.

5 Achenbach TM, Edelbrock CS. The classification of child psychopathology: a review and analysis of empirical efforts. Psychol Bull 1978; 85: 1275-1301.

6 Achenbach TM. The classification of children's psychiatric symptoms: a factor-analytic study. Psychol Monogr General Appl 1966; 80: 1-37.

7 Deighton J, Tymms P, Vostanis P, Belsky J, Fonagy P, Brown A, et al. The development of a school-based measure of child mental health. J Psychoeduc Assess 2013; 31: 247-57.

8 Achenbach TM, Rescorla LA. Manual for the ASEBA School-Age Forms \& Profiles. University of Vermont Research Center for Children, Youth and Families, 2001.

9 Martel MM. Sexual selection and sex differences in the prevalence of childhood externalizing and adolescent internalizing disorders. Psychol Bull 2013; 139: 1221-59.

10 Green H, McGinnity A, Meltzer H, Ford T, Goodman R. Mental Health of Children and Young People in Great Britain, 2004. Palgrave Macmillan, 2005

11 Wolpert M, Deighton J, Patalay P, Martin A, Fitzgerald-Yau N, Demir E, et al. Me and My School: Findings from the National Evaluation of Targeted Mental Health in Schools. DFE, 2011 (https://www.gov.uk/government/uploads/ system/uploads/attachment_data/file/184060/DFE-RR177.pdf).

12 Hobbs G, Vignoles A. Is children's free school meal 'eligibility' a good proxy for family income? Br Educ Res J 2010; 36: 673-90.

13 Department for Children, Schools and Families. Schools, Pupils and Their Characteristics. Department for Children, Schools and Families, 2009 (http://webarchive.nationalarchives.gov.uk/20120504203418/http:// education.gov.uk/rsgateway/DB/SFR/s000843/index.shtml).

14 Patalay $P$, Deighton J, Fonagy $P$, Vostanic $P$, Wolpert $M$. Clinical validity of the Me and My School questionnaire: a self-report mental health measure for children and adolescents. Child Adol Psychiat Mental Health 11 Jun 2014 (doi: 10.1186/1753-2000-8-17). 
15 Goodman R, Meltzer H, Bailey V. The strengths and difficulties questionnaire: a pilot study on the validity of the self-report version. Eur Child Adol Psych 1998; 7: 125-30.

16 DfE. Statistical First Release SFR 18/2011. Department for Education, 2011.

17 Brunner M, Nagy G, Wilhelm O. A tutorial on hierarchically structured constructs. J Pers 2012; 80: 796-846.

18 Muthén LK, Muthén BO. Mplus User's Guide. (7th edn). Muthén \& Muthén, 2012.

19 Finney SJ, DiStefano C. Non-normal and categorical data in structural equation modeling. In Structural Equation Modeling: A Second Course (eds GR Hancock, RO Mueller): 269-314. Information Age, 2006.

$20 \mathrm{Hu}$ L-T, Bentler PM. Cutoff criteria for fit indexes in covariance structure analysis: conventional criteria versus new alternatives. Struct Equ Modeling 1999; 6: 1-55.

21 Yung Y-F, Thissen D, McLeod L. On the relationship between the higher-orde factor model and the hierarchical factor model. Psychometrika 1999; 64: 113-28

22 Chen $\mathrm{H}$, Cohen $\mathrm{P}$, Chen S. How big is a big odds ratio? Interpreting the magnitudes of odds ratios in epidemiological studies. Commun Stat Simul Comput 2010; 39: 860-4

23 Masten AS, Roisman GI, Long JD, Burt KB, Obradovic J, Riley JR, et al. Developmental cascades: linking academic achievement and externalizing and internalizing symptoms over 20 years. Dev Psych 2005; 41: 733-46.
24 Moffitt TE, Caspi A, Dickson N, Silva P, Stanton W. Childhood-onset versus adolescent-onset antisocial conduct problems in males: natural history from ages 3 to 18 years. Dev Psychopathol 2009; 8: 399.

25 Nolen-Hoeksema S, Girgus JS. The emergence of gender differences in depression during adolescence. Psychol Bull 1994; 115: 424-43.

26 Goodman A, Lamping DL, Ploubidis GB. When to use broader internalising and externalising subscales instead of the hypothesised five subscales on the strengths and difficulties questionnaire (SDQ): data from British parents, teachers and children. J Abnormal Child Psych 2010; 38: 1179-91.

27 De Los Reyes A. Strategic objectives for improving understanding of informant discrepancies in developmental psychopathology research. Dev Psychopathol 2013; 25: 669-82.

28 Lahey BB, Van Hulle CA, Singh AL, Waldman ID, Rathouz PJ. Higher-order genetic and environmental structure of prevalent forms of child and adolescent psychopathology. Arch Gen Psychiatry 2011; 68: 181-9.

29 Cross-Disorder Group of the Psychiatric Genomics Consortium. Identification of risk loci with shared effects on five major psychiatric disorders: a genome-wide analysis. Lancet 2013; 381: 1371-9.

30 Fernandez J. Immune diosrders. In Merck Manual Online (eds RS Porter, JL Kaplan): Merck Sharp \& Dohme, 2014

31 Moffitt TE, Harrington HL, Caspi A, Kim-Cohen J, Goldberg D, Gregory AM, et al. Depression and generalised anxiety disorder: cumulative and sequential co-morbidity in a birth cohort followed to age 32. Arch Gen Psychiatry 2007: 64: $651-60$.

\title{
Did you mean to be so rude? Meaningless comments and the Mental State Examination
}

\author{
Suhanthini Farrell
}

Appearance and behaviour: Middle-aged woman, morbidly obese, frizzy greying hair, dressed inappropriately.

'There,' she said, pointing with a trembling finger to her copy of an old clinic letter, as she blinked back tears. 'Would you come back if someone wrote that about you?'

Probably not. Like her, I would have been horrified and humiliated, and hidden away from mental health services until it was almost too late. The fact that it was technically true made no difference.

I reflected on what that sentence really added to her clinical care. Her age was clear from her date of birth; her weight, if relevant, should have been recorded as part of her medical history. The frizziness or otherwise of a person's hair has no influence on my prescribing practice, and the comment on the 'inappropriateness' of her dress was sufficiently vague to add nothing to my understanding while being really quite offensive.

Psychiatric records are filled with comments and descriptions just like this. I began to wonder why. Of course there are times when a person's appearance will be clinically relevant and require comment; just as a cardiologist would note a patient's xanthelasma, or a neurologist their shuffling gait. But how often do we actually think about how useful the information is? Or do we just describe the patient's appearance by rote because we were told, as medical students, that it's just what psychiatrists do?

Surely the Mental State Examination should be just that - an examination that critically evaluates the evidence in front of us, using our clinical skills and experience, ultimately adding value to an assessment. A collection of random, casual comments adds nothing, as well as (in our modern world of transparency and copied clinic letters) having the potential to cause harm.

Perhaps we could start by taking a few seconds before we put pen to paper - or mouth to voice recorder - and think about what we actually need to say. 